\title{
Effect of ST36 on Body Weight and Blood Lipid Concentration in Obese Animal Models: a Protocol of Systematic Review and Meta-analysis
}

\section{Zepeng Zhang}

Changchun University of Chinese Medicine

Haipeng Huang

Changchun University of Chinese Medicine

\section{Xingquan Wu}

Changchun University of Chinese Medicine

Di Jin

Changchun University of Chinese Medicine

Xiaoru Xu

Changchun University of Chinese Medicine

Xiangyan Li

Changchun University of Chinese Medicine

Zhihong Wang ( $\nabla$ wzh6172555@163.com )

Changchun University of Chinese Medicine https://orcid.org/0000-0001-7043-0733

\section{Protocol}

Keywords: ST 36, body weight, blood lipid concentration, obese animal models

Posted Date: February 16th, 2021

DOl: https://doi.org/10.21203/rs.3.rs-207163/v1

License: (c) (1) This work is licensed under a Creative Commons Attribution 4.0 International License. Read Full License 


\section{Abstract}

Background: Obesity is a health crisis for people all over the world. ST 36 plays an irreplaceable role in the treatment of weight control and blood lipid in traditional Chinese medicine. The aim of this systematic review is to assess and synthesis evidence of efficacy of ST 36 for body weight and blood lipid concentration.

Methods: We will search studies on the anti-obesity effect of ST36 in animal models from the following databases in consultation with an experienced medical information-scientist or librarian: PubMed, Embase, Web of Science, and Scopus. Searches were performed until January 2021. There is no time of publication limitation. The outcomes will be measured with body weight and outcomes related to blood lipid level. SYRCLE Risk tool for animal studies will be conducted to assess the risk of bias. The protocol will be conducted according to approach and Preferred Reporting Items for Systematic Review and MetaAnalysis Protocols 2015.

Discussion: This systematic review will bring the following results: 1) whether ST 36 acupoint is closely related to the intervention of obesity and blood lipid, 2) If the ST 36 therapy have the function of reducing weight and fat, which intervention means is the best choice, 3) Massage is a simple and easy to learn intervention method, and can treat ST36 by patients themselves, it is called Dao yin in Chinese.

\section{PROSPERO registration number: CRD42020177223}

\section{Background}

Obesity has been classified as one of the top five risk factors for death and disability[1]. Although national health awareness has increased year by year, the prevalence of obesity in more than 70 countries has doubled since 1980[2]. Metabolic disorders accompanied by obesity are the main causes of many high-risk diseases such as cardiovascular and cerebrovascular diseases, diabetes, and kidney diseases[3] Among the interventions that have been promoted so far, drug treatment and surgical treatment are at risk and have a poor prognosis. However, the efficacy of simple lifestyle intervention is limited, and the incidence of obesity has not been significantly improved[4].

Traditional Chinese medicine has maintained the human health in China for thousands of years, and has accumulated rich experience in the treatment of various diseases[5]. Several clinical and trials provide evidence of TCM's intervention on obesity[6, 7] Acupoint therapies like massage, acupuncture, electroacupuncture and acupoint injection are particularly suitable for obese patients because it is economical, simple and safe[8, 9]. In these treatment protocols, Zusanli (ST36), Zhongwan (CV12), Sanyinjiao (SP6), Neiting (ST44), Yanglingquan (GB34), and Fenglong (ST 40)selected as acupoints, among which ST36 is the most commonly used acupoint $[10,11]$. These studies indicate that ST36 is a commonly used acupoint therapy for obesity $[12,13]$. 
Body weight and blood lipid concentration are important indicators to measure the therapeutic effect on obesity[14]. Clinical studies have reported ST36 acupoint therapy could reduce body weight and blood lipid concentration[15], which also showed that ST36 is an effective treatment target for obesity. Several animal studies have reported the anti-obesity effect of ST36, but reported only descriptive outcomes and mechanism without quantitatively analyzing the data[16]. We found several existing meta-analyses, but they all analyze the effects of acupuncture or electro-acupuncture on obesity, and there is no systematic review of ST36 $[17,18]$.

Therefore, a high-quality systematic review and meta-analysis to summarize current clinical evidence is urgently needed. Thus, this systematic review aims to assess the efficacy of ST36 as an adjuvant treatment for reducing body weight and blood lipid concentration in obese animal models.

\section{Methods}

The systematic review was performed in accordance with Preferred Reporting Items for Systematic reviews and Meta-Analyses (PRISMA) guidelines[19].

\subsection{Study registration}

The protocol of this systematic review and meta-analysis has been registered in the International Prospective Register of Systematic Reviews (PROSPERO), and the registration number is CRD42020177223. Ethical approval is not required for this study.

\subsection{Inclusion and exclusion criteria 2.2.1. Types of studies}

This research will include only animal research articles which evaluated the effects of ST36 on the obese animal model with controlled studies.

\subsubsection{Types of animal models}

All studies used an obesity animal model which accompany with body weight and blood lipid level change will be included in this review.

\subsubsection{Types of interventions}

The conventional interventions with ST36 acupoint, including acupuncture, acupoint catgut, massage, acupressure, electro-acupuncture.

\subsubsection{Types of controls}

Another intervention or a non-exposed control group like sham-acupuncture.

\subsubsection{Outcomes.}


Body weight and outcomes related to blood lipid level as outcomes. There will be no limit about the measurement of results.

\subsection{Study search}

We identified studies on the anti-obesity effect of ST36 in animal models from the following databases in consultation with an experienced medical information-scientist or librarian: PubMed, Embase, Web of Science, and Scopus. Searches were performed until January 2021. The search terms used were sufficiently broad to capture the majority of the published data using ST36 to treat obese animal models. The search terms used were (obese OR obesity OR body weight OR blood lipid concentration OR antiobesity OR Bariatrics) AND (ST36 OR zusanli). There was no language restriction. A search strategy for PubMed was developed with guidance. Animal filters validated for PubMed/MEDLINE and Embase were used to enhance the search efficiency with respect to identifying all animal studies.

\subsection{Study selection}

One investigator will search studies from those databases and list titles and abstract of all articles. Two reviewers will read the title and abstract of all articles and select eligible articles independently according to inclusion and exclusion criteria. Reviewers will then download articles and read full text when decisions cannot be made according to titles and abstract. A final decision will be made through consensus when there are discrepancies between researchers. Disagreement in this and all following steps of the systematic review process will be resolved by discussion or adjudication by a third reviewer, when necessary. The flow diagram of study selection was shown in Fig. 1.

\subsubsection{Prioritise the exclusion criteria}

1. Not an animal study.

2. Not an obesity animal model.

3. Not treatment with ST36 acupoint.

4. Not case studies, cross-over studies, studies without a separate control group.

5. Not an original full research paper (e.g. review, editorial).

6. Combined with other special therapies in control group alone.

7. No relevant outcome measure reported (e.g. Mechanisms Research only).

8. Data could not be extracted.

9. There is no restriction on the language, publication dates and publication status of studies.

10. All other outcomes that are not directly related to body weight and blood lipid.

\subsection{Data extraction and management}

Two reviewers will independently extract data from each article. We first try to extract numerical data from tables, text or figures. If these are not reported, we will extract data from graphs using digital ruler software. In case data are not reported or unclear, we will attempt to contact authors by e-mail (max. 2 
attempts). In case an outcome is measured at multiple time points, data from the time point where efficacy is highest will be included.

\subsection{Risk of bias assessment}

Risk of bias was assessed independently by two investigators per the 2014 SYRCLE Risk tool for animal studies. We will take nine categories to evaluate bias related to selection, performance, detection, attrition and reporting. Risk of bias is judges as low unclear, or high in following domains: peer reviewed publication, control of temperature, random allocation to treatment or control, blinded induction of model, blinded assessment of outcome, animal model (aged, gender), sample size calculation, compliance with animal welfare regulations, statement of potential conflict of interests.

\subsection{Strategy for data synthesis}

RevMan 5.3 will be used to conduct meta-analysis. Meta-analysis will be performed for outcome measures reported in more than 8 studies. If meta-analysis is impossible, data will be reported by a descriptive summary. We consider all outcomes as continuous data. All data will be analyzed by standardized mean difference or mean difference with $95 \% \mathrm{Cls}$. Because of the exploratory nature of animal studies, a random effects model will be used to account for anticipated heterogeneity.

\subsection{Investigation of heterogeneity}

Heterogeneity between the studies will be assessed using the $I^{2}$ statistic. If $I^{2}>50 \%$, we considered significant heterogeneity to be present.

\subsection{Analysis of subgroups or subsets}

If necessary, we will conduct a subgroup analysis of animal age and gender, time of different modeling methods.

\subsection{Sensitivity analysis}

The sensitivity analysis will be used to assess the effect of each study on the random effects model. The sensitivity of the general combined effect of all outcome indicators is analyzed by the exclusion method, that is, each study is excluded, and the remaining studies will be re-analyzed to identify the stability of the results.

\subsection{Publication bias}

For meta-analyses using the mean difference or odds ration as effect measure and containing at least 10 studies, we will produce funnel plots and assess publication bias using Egger's regression test.

\subsection{Patient and public involvement}


No patient or public will be involved in our study directly. We only use data that existed in studies published.

\subsection{Ethics and dissemination}

There is no need of ethical approval in this study, because there is nothing of the data which has a relationship with an individual patient. We will complete this systematic review according to the PRISMA guidelines. The review will provide an assessment of effect and ST 36 for body weight and blood lipid concentration in obese animal modelse. We will publish the findings in a peer-reviewed, open assess journal and the finished systematic review and meta-analysis will be disseminated online, which would be obtained freely for anyone.

\section{Discussion}

Obesity and hyperlipidemia are great threats to human health, which can induce many kinds of chronic diseases[20]. Traditional Chinese Medicine therapy, such as acupuncture and massage, has the advantages of safety and easy operation [21]. ST36 acupoint is also a common acupoint to regulate body weight and blood lipid [22]. Combined with the physiological position of ST 36, it is particularly safe and convenient to treat it [23]. However, there is no systematic review to clarify whether ST36 acupoint has a definite effect on obesity and blood lipid.

This systematic review will bring the following results: 1) whether ST 36 acupoint is closely related to the intervention of obesity and blood lipid, 2) If the ST 36 therapy have the function of reducing weight and fat, which intervention means is the best choice, 3 ) Massage is a simple and easy to learn intervention method, and can treat ST36 by patients themselves, it is called Dao yin in Chinese [24, 25]. If ST36 has significant effect, it will provide a simple and effective way to lose weight for everyone.

Limitations: 1) This program is only a systematic review of preclinical research, and it cannot provide reliable evidence for clinical application. 2) Some publication in Chinese will be included, the efficacy of therapy might be overestimated due to publication bias.

\section{Abbreviations}

ST 36: Zusanli, PRISMA: Preferred Reporting Items for Systematic reviews and Meta-Analyses, PROSPERO: the International Prospective Register of Systematic Reviews.

\section{Declarations}

\section{Ethics approval and consent to participate}

Not applicable 


\section{Consent for publication}

Not applicable

\section{Availability of data and materials}

The datasets used and/or analysed during the current study are available from the corresponding author on reasonable request.

\section{Authors' contributions}

$\mathrm{XL}$ and $\mathrm{ZW}$ conceived and designed the protocol. $\mathrm{ZZ}$ and $\mathrm{XX}$ registered the protocol review in the PROSPERO database and drafted the manuscript. $\mathrm{HH}$ and ZZ designed the search strategy. ZZ and ZW drafted the protocol. All authors contributed to and approved the final manuscript of the protocol review.

\section{Funding}

This article is supported by the National Key Research and Development Program of China (2019YFC1709901), Sanming Project of Medicine in Shenzhen (201802074), the Science and Technology Development Plan Project of Jilin Province (20190101010JH)

\section{Competing interests}

None declared.

\section{References}

1. LM J, S V, A P, CJ M, C W, F I, et al.: The obesity transition: Stages of the global epidemic. The lancet Diabetes \& endocrinology 2019;7:231-240.

2. Afshin A, Forouzanfar MH, Reitsma MB, Sur P, Estep K, Lee A, et al.: Health effects of overweight and obesity in 195 countries over 25 years. The New England journal of medicine 2017;377:13-27.

3. Roden M, Shulman Gl: The integrative biology of type 2 diabetes. Nature 2019;576:51-60.

4. Reilly SM, Saltiel AR: Adapting to obesity with adipose tissue inflammation. Nature reviews Endocrinology 2017;13:633-643.

5. Xu L, Zhao W, Wang D, Ma X: Chinese medicine in the battle against obesity and metabolic diseases. Frontiers in physiology 2018;9:850.

6. Sang T-T, Guo C-J, Guo D-D, Wang X-Y: [effect of traditional chinese medicine in inhibiting obesity and inflammatory diseases by regulating gut microbiota]. Zhongguo Zhong yao za zhi = Zhongguo zhongyao zazhi $=$ China journal of Chinese materia medica 2018;43:3235-3242.

7. Di S, Wang Y, Han L, Bao Q, Gao Z, Wang Q, et al.: The intervention effect of traditional chinese medicine on the intestinal flora and its metabolites in glycolipid metabolic disorders. Evidence-based complementary and alternative medicine : eCAM 2019;2019:2958920. 
8. Zhang R-Q, Tan J, Li F-Y, Ma Y-H, Han L-X, Yang X-L: Acupuncture for the treatment of obesity in adults: A systematic review and meta-analysis. Postgrad Med J 2017;93:743-751.

9. Wang F, Tian D-R, Han J-S: Electroacupuncture in the treatment of obesity. Neurochem Res 2008;33:2023-2027.

10. Zhao M, Liu Z, Su J: The time-effect relationship of central action in acupuncture treatment for weight reduction. J Tradit Chin Med 2000;20:26-29.

11. Tian D-R, Li X-D, Wang F, Niu D-B, He Q-H, Li Y-S, et al.: Up-regulation of the expression of cocaine and amphetamine-regulated transcript peptide by electroacupuncture in the arcuate nucleus of dietinduced obese rats. Neuroscience letters 2005;383:17-21.

12. Lai $F$, Ren $Y$, Lai $C$, Chen $R$, Yin $X$, Tan $C$, et al.: Acupuncture at zusanli (st36) for experimental sepsis: A systematic review. Evidence-based complementary and alternative medicine : eCAM 2020;2020:3620741.

13. Fu H, Chen B, Hong S, Guo Y: Acupuncture therapy for the treatment of myelosuppression after chemotherapy: A literature review over the past 10 years. J Acupunct Meridian Stud 2015;8:122-126.

14. Herrera $\mathrm{E}$, Ortega-Senovilla $\mathrm{H}$ : Implications of lipids in neonatal body weight and fat mass in gestational diabetic mothers and non-diabetic controls. Curr Diab Rep 2018;18:7.

15. Belivani M, Dimitroula C, Katsiki N, Apostolopoulou M, Cummings M, Hatzitolios Al: Acupuncture in the treatment of obesity: A narrative review of the literature. Acupunct Med 2013;31:88-97.

16. Ji B, Hu J, Ma S: Effects of electroacupuncture zusanli (st36) on food intake and expression of pomc and trpv1 through afferents-medulla pathway in obese prone rats. Peptides 2013;40:188-194.

17. Sui Y, Zhao HL, Wong VCW, Brown N, Li XL, Kwan AKL, et al.: A systematic review on use of chinese medicine and acupuncture for treatment of obesity. Obesity reviews : an official journal of the International Association for the Study of Obesity 2012;13:409-430.

18. Sheng J, Jin X, Zhu J, Chen Y, Liu X: The effectiveness of acupoint catgut embedding therapy for abdominal obesity: A systematic review and meta-analysis. Evidence-based complementary and alternative medicine : eCAM 2019;2019:9714313.

19. Liberati A, Altman DG, Tetzlaff J, Mulrow C, Gøtzsche PC, loannidis JP, et al.: The prisma statement for reporting systematic reviews and meta-analyses of studies that evaluate healthcare interventions: Explanation and elaboration. BMJ (Clinical research ed) 2009;339:b2700.

20. Vekic J, Zeljkovic A, Stefanovic A, Jelic-Ivanovic Z, Spasojevic-Kalimanovska V: Obesity and dyslipidemia. Metabolism: clinical and experimental 2019;92:71-81.

21. Tao WW, Jiang H, Tao XM, Jiang P, Sha LY, Sun XC: Effects of acupuncture, tuina, tai chi, qigong, and traditional chinese medicine five-element music therapy on symptom management and quality of life for cancer patients: A meta-analysis. Journal of pain and symptom management 2016;51:728-747.

22. Lan D, Xu N, Sun J, Li Z, Liao R, Zhang H, et al.: Electroacupuncture mitigates endothelial dysfunction via effects on the pi3k/akt signalling pathway in high fat diet-induced insulin-resistant rats. Acupunct Med 2018;36:162-169. 
23. Wang LQ, Chen Z, Zhang K, Liang N, Yang GY, Lai L, et al.: Zusanli (st36) acupoint injection for diabetic peripheral neuropathy: A systematic review of randomized controlled trials. Journal of alternative and complementary medicine (New York, NY) 2018;24:1138-1149.

24. Schlaeger JM, Gabzdyl EM, Bussell JL, Takakura N, Yajima H, Takayama M, et al.: Acupuncture and acupressure in labor. Journal of midwifery \& women's health 2017;62:12-28.

25. Zhang HL, Li JS, Yu XQ, Li SY, Halmurat U, Xie Y, et al.: An evaluation of activity tolerance, patientreported outcomes and satisfaction with the effectiveness of pulmonary daoyin on patients with chronic obstructive pulmonary disease. International journal of chronic obstructive pulmonary disease 2017;12:2333-2342.

\section{Figures}




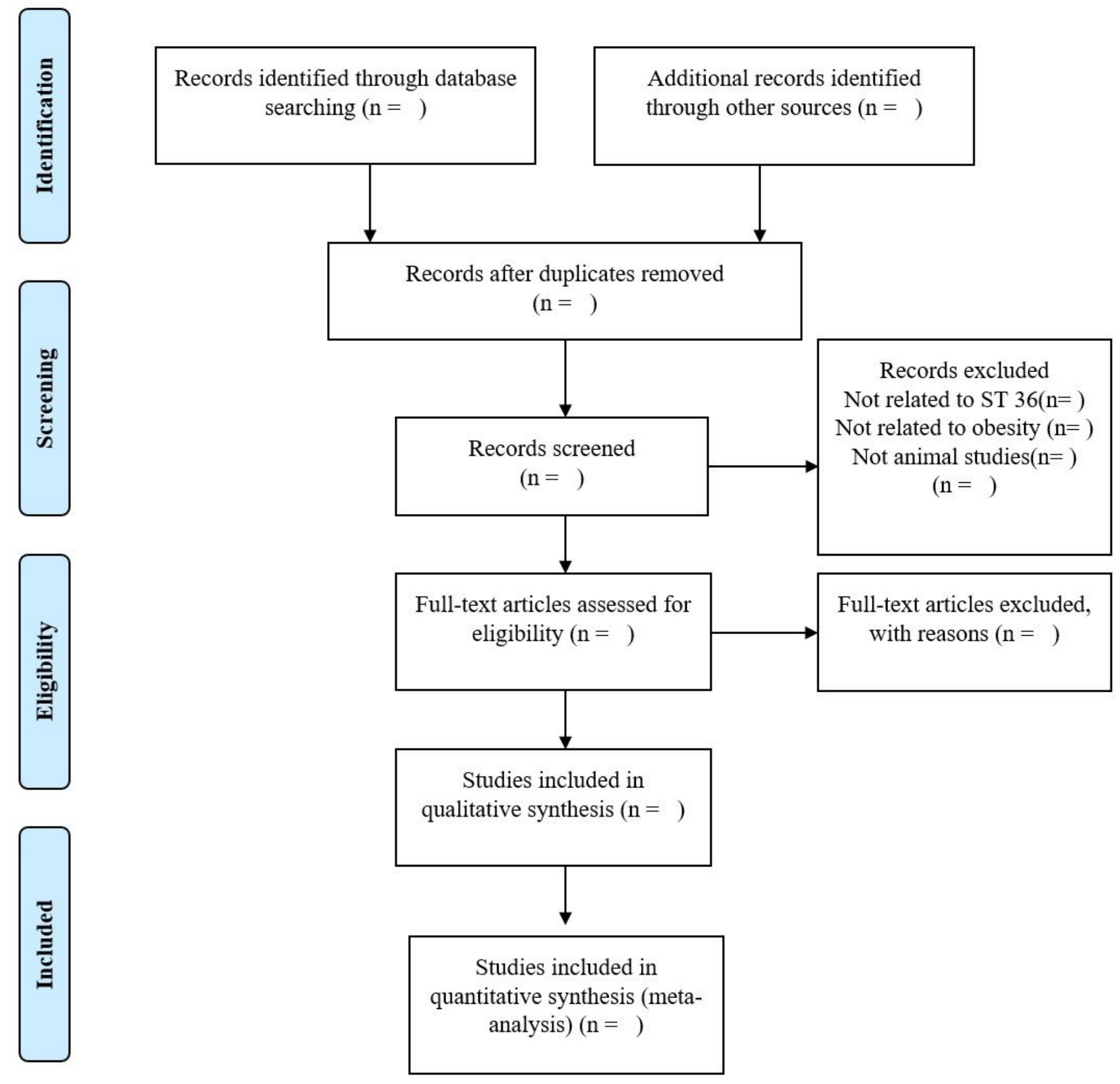

Figure 1

Flow chart of study selection.

\section{Supplementary Files}

This is a list of supplementary files associated with this preprint. Click to download.

- PRISMAPchecklist.doc 